\title{
Algebraic flows on abelian varieties
}

\author{
By Emmanuel Ullmo at Bures-sur-Yvette and Andrei Yafaev at London
}

\begin{abstract}
Let $A$ be an abelian variety. The abelian Ax-Lindemann theorem shows that the Zariski closure of an algebraic flow in $A$ is a translate of an abelian subvariety of $A$. The paper discusses some conjectures on the usual topological closure of an algebraic flow in $A$. The main result is a proof of these conjectures when the algebraic flow is given by an algebraic curve.
\end{abstract}

\section{Introduction}

The purpose of this paper is to study algebraic flows on abelian varieties. We formulate two (interrelated) conjectures, prove a rather general statement concerning these conjectures and provide examples where our statement applies. Let $A$ be a complex abelian variety of dimension $g$. Let $\Gamma$ be a lattice in $\mathbb{C}^{g}$ such that $A \simeq \mathbb{C}^{g} / \Gamma$ and let

$$
\pi: \mathbb{C}^{g} \rightarrow A \simeq \mathbb{C}^{g} / \Gamma
$$

be the uniformizing map. A complex algebraic subvariety $Z$ of $A$ is said to be weakly special if $Z=P+B$ for an abelian subvariety $B$ and a point $P$ of $A$. If moreover $P$ is a torsion point, then $Z=B+P$ is said to be special.

The following abelian version of Ax-Lindemann theorem is due to Ax (see [1] and [2]) and plays an important role in the new proof by Pila and Zannier of the Manin-Mumford conjecture [6]. Note that the paper [6] provides a different proof of the abelian Ax-Lindemann theorem. Yet another proof was given by Orr (see [4]).

Theorem 1.1 (Abelian Ax-Lindemann). Let $\Theta$ be a complex algebraic subvariety of $\mathbb{C}^{g}$. Then any component of the Zariski closure of $\pi(\Theta)$ is weakly special.

In this paper, instead of looking at the Zariski closure of $\pi(\Theta)$, we attempt to characterise the usual topological closure $\overline{\pi(\Theta)}$ of $\pi(\Theta)$.

Let $W \subset \mathbb{C}^{g}$ be a $\mathbb{R}$-vector space such that $\Gamma_{W}:=\Gamma \cap W$ is a lattice in $W$. Then $W / \Gamma_{W}$ is a real torus and is a closed real analytic subset of $A$. A real analytic subvariety $Z$ of $A$ is

The second author is very grateful to the ERC (Grant No. ERC 511343) for financial support. 
said to be real weakly special if $Z=P+W / \Gamma_{W}$ for a point $P$ and a real subtorus $W / \Gamma_{W}$ of $A$. A real affine subspace of $\mathbb{C}^{g} \simeq \mathbb{R}^{2 g}$ of the form $W+Q$ where $Q$ is a point in $\mathbb{C}^{g}$ and $W$ is a $\mathbb{R}$-vector subspace of $\mathbb{C}^{g} \simeq \mathbb{R}^{2 g}$ is said to be real weakly pre-special if $\pi(Q)+\pi(W)$ is real weakly special. By definition, $Q+W$ is real weakly pre-special if and only if $W \cap \Gamma$ is a lattice in $W$.

We will study the following conjecture.

Conjecture 1.2. Let $A$ be a abelian variety of dimension $g$. Let $\Theta$ be a complex irreducible algebraic subvariety of $\mathbb{C}^{g}$. Then there exists a finite number $Z_{1}, \ldots, Z_{r}$ of real weakly special subvarieties of $A$ such that

$$
\overline{\pi(\Theta)}=\pi(\Theta) \cup \bigcup_{k=1}^{r} Z_{k} .
$$

Let $Z=W / \Gamma_{W}$ be a real subtorus of $A$. Then the normalized Lebesgue measure on $W$ induces a canonical probability measure $\mu_{Z}$ on $A$. The support $\operatorname{Supp}\left(\mu_{Z}\right)$ of $\mu_{Z}$ is $Z$. Using the description of a real weakly special subvariety $Z$ of $A$ of the form $Z=P+W / \Gamma_{W}$, we see that we have also a canonical probability measure $\mu_{Z}$ on $A$ such that $\operatorname{Supp}\left(\mu_{Z}\right)=Z$.

Let $\omega$ be the canonical translation invariant positive $(1,1)$-form on $\mathbb{C}^{g}$. We fix a $\mathbb{C}$-basis of $\mathbb{C}^{g}$ with associated complex coordinates $\left(z_{1}, \ldots, z_{g}\right)$ with $z_{k}=x_{k}+i y_{k}$ for $1 \leq k \leq g$. Then we may choose

$$
\omega=\frac{i}{2} \sum_{k=1}^{g} d z_{k} \wedge d \bar{z}_{k}=\sum_{k=1}^{g} d x_{k} \wedge d y_{k} .
$$

For $R>0$, we let $B(0, R)=\left\{\left(z_{1}, \ldots, z_{g}\right) \in \mathbb{C}^{g}:\left|z_{k}\right|<R\right\}$.

By slight abuse of notations we will often identify functions on $A$ with $\Gamma$-invariant functions on $\mathbb{C}^{g}$.

Assume that $\Theta$ is an algebraic subvariety of $\mathbb{C}^{g}$ of dimension $d$. Define, for all $R$ big enough, the probability measure $\mu_{\Theta, R}$ on $\mathbb{C}^{g}$ such that for any continuous function $f$ on $\mathbb{C}^{g}$,

$$
\mu_{\Theta, R}(f)=\frac{1}{V_{R}} \int_{\Theta \cap B(0, R)} f \omega^{d},
$$

where $V_{R}=\int_{\Theta \cap B(0, R)} \omega^{d}$.

The pushforward of $\mu_{\Theta, R}$ (that we will still, by abuse of notation denote $\mu_{\Theta, R}$ ) to $A$ induces a probability measure on $A$ with support contained in $\overline{\pi(\Theta)}$.

The measure theoretical version of the topological Conjecture 1.2 is

Conjecture 1.3. Let $A$ be a abelian variety of dimension $g$. Let $\Theta$ be an irreducible complex algebraic subvariety of $\mathbb{C}^{g}$. Then there exists a finite number of real weakly special subvarieties $Z_{1}, \ldots, Z_{r}$ and some positive real numbers $c_{1}, \ldots, c_{r}$ such that $\mu_{\Theta, R}$ converges weakly to the measure $\sum_{k=1}^{r} c_{k} \mu_{Z_{k}}$. This means that for any continuous function $f$ on $A$ we have

$$
\mu_{\Theta, R}(f) \rightarrow \sum_{k=1}^{r} c_{k} \mu_{Z_{k}}(f)
$$

as $R \rightarrow \infty$. 
The main result of this paper is the proof of these conjectures for the image $\pi(C)$ of a curve $C$ in $\mathbb{C}^{g}$. In this case, the real weakly special subvarieties $Z_{1}, \ldots, Z_{r}$ appearing in the statements of Conjectures 1.2 and 1.3 are described simply in terms of $\Gamma$ and the geometry at infinity of $C$. The precise version of this result is given in the statement of Theorem 2.4.

In Sections 2 and 3 we define the Mumford-Tate torus of an algebraic subvariety of $\mathbb{C}^{g}$. We also define the asymptotic Mumford-Tate torus of an infinite branch of a curve contained in $\mathbb{C}^{g}$. We prove a certain number of properties of these objects that we use later on. We then review characters of these Mumford-Tate tori and the Weyl criterion. Section 4, which is the main technical part of the proof of Theorem 2.4, concerns itself with the estimation of some oscillatory integrals. After proving the main result in Section 5, we provide enlightening examples where the conclusion of Conjecture 1.2 holds.

The hyperbolic version of the Ax-Lindemann theorem for Shimura varieties had been proven (see [8], [5] and[3]). Conjectures 1.2 and 1.3 have natural and interesting formulations in this context. These questions will be studied in further works.

Acknowledgement. We are extremely grateful to James Wright who provided very detailed answers to our questions regarding oscillatory integrals. We would like to extend our thanks to the referee for his careful reading and his detailed comments which helped us improve the presentation of the paper.

\section{Mumford-Tate and asymptotic Mumford-Tate tori}

2.1. Mumford-Tate tori. Let $\Theta$ be an irreducible algebraic subvariety of $\mathbb{C}^{g}$ containing the origin $O$ of $\mathbb{C}^{g}$. The Mumford-Tate group $\mathrm{MT}(\Theta)$ of $\Theta$ is defined as the smallest $\mathbb{Q}$-vector subspace $W$ of $\Gamma \otimes \mathbb{Q}$ such that $\Theta \subset W \otimes \mathbb{R}$. More generally, let $\Theta$ be an irreducible algebraic subvariety of $\mathbb{C}^{g}$ and $P \in \Theta$. Then we define $\operatorname{MT}(\Theta)$ as $\operatorname{MT}(\Theta-P)$. One can check that the definition is independent of the choice of $P \in \Theta$. Let $W_{\Theta}:=\operatorname{MT}(\Theta) \otimes \mathbb{R}$ and $\mathbb{T}_{\Theta}=W_{\Theta} /\left(W_{\Theta} \cap \Gamma\right)$. We denote by $\mathbb{T}_{\Theta}^{\prime}$ the real weakly-special subvariety of $A$,

$$
\mathbb{T}_{\Theta}^{\prime}=\pi(P)+\mathbb{T}_{\Theta} .
$$

Then $\mathbb{T}_{\Theta}^{\prime}$ is independent of $P$ and $\mathbb{T}_{\Theta}^{\prime}$ is the smallest real weakly special subvariety of $A$ containing $\pi(\Theta)$. Then we have the first natural upper bound for the topological closure of $\pi(\Theta)$ in $A$,

$$
\overline{\pi(\Theta)} \subset \mathbb{T}_{\Theta}^{\prime}
$$

2.2. Asymptotic Mumford-Tate tori for curves. Let $C$ be a curve in $\mathbb{C}^{g}$. Let $C^{*}$ be the Zariski closure of $C$ in $\mathbb{P}^{1}(\mathbb{C})^{g}$. Then $C^{*}-C$ is a finite set of points $\left\{P_{1}, \ldots, P_{s}\right\}$. For each $i \in\{1, \ldots, s\}$ we can fix a neighbourhood $U_{i}$ of $P_{i}$ such that the connected components of $C \cap U_{i}$ have a parametrization by power series. A infinite branch $C_{\alpha}$ of $C$ near a point at infinity $P_{i}$ is a complex analytic connected component of $C \cap U_{i}$. We assume that for all $i$ and $j$ we have $U_{i} \cap U_{j}=\emptyset$.

Let $C_{\alpha}$ be such a branch. Let $\left(z_{1}, \ldots, z_{g}\right)$ be the coordinates on $\mathbb{C}^{g}$. Then there exists at least one coordinate $z=z_{i}$ which is unbounded when $\underline{Z}=\left(z_{1}, \ldots, z_{g}\right)$ varies in $C_{\alpha}$.

We say that a real weakly pre-special subvariety $Q+W$ is asymptotic to $C_{\alpha}$ if the Euclidean distance from a point $\underline{Z}=\left(z_{1}, \ldots, z_{g}\right)$ of $C_{\alpha}$ to $Q+W$ tends to 0 as $z=z_{i}$ tends 
to $\infty$. Note that by definition if $P$ is a point of $C$, then $P+\operatorname{MT}(C) \otimes \mathbb{R}$ is asymptotic to $C_{\alpha}$ for all infinite branches $C_{\alpha}$ of $C$.

Lemma 2.1. An intersection of two real weakly pre-special subvarieties which are asymptotic to $C_{\alpha}$ is real weakly pre-special and asymptotic to $C_{\alpha}$.

Proof. Let $P+W$ and $Q+W^{\prime}$ be two real weakly pre-special subvarieties which are asymptotic to $C_{\alpha}$. Then the distance between $P+W$ and $Q+W^{\prime}$ is zero. Therefore $(P+W) \cap\left(Q+W^{\prime}\right)$ is not empty. Let $R \in(P+W) \cap\left(Q+W^{\prime}\right)$. Then $P+W=R+W$ and $Q+W^{\prime}=R+W^{\prime}$. As a consequence,

$$
(P+W) \cap\left(Q+W^{\prime}\right)=R+W \cap W^{\prime} .
$$

The lattice $\Gamma$ defines a $\mathbb{Q}$-structure on $\mathbb{C}^{g} \simeq \mathbb{R}^{2 g}$. By definition, $W$ and $W^{\prime}$ are defined over $\mathbb{Q}$ for this $\mathbb{Q}$-structure. Therefore $W \cap W^{\prime}$ is defined over $\mathbb{Q}$ and $R+W \cap W^{\prime}$ is real weakly prespecial. Let $\underline{Z}_{n}$ be a sequence of points of $C_{\alpha}$ tending to the point at infinity. Then there exist $P_{n} \in W, Q_{n} \in W^{\prime}$ and two sequences $\epsilon_{n}$ and $\epsilon_{n}^{\prime}$ of points in $\mathbb{C}^{g}$ tending to $O$ such that

$$
\underline{Z}_{n}=R+P_{n}+\epsilon_{n}=R+Q_{n}+\epsilon_{n}^{\prime} .
$$

Therefore we have $\left\|P_{n}\right\| \rightarrow \infty$ and $d\left(P_{n}, W^{\prime}\right) \rightarrow 0$ as $n \rightarrow \infty$. It follows that

$$
\lim _{n \rightarrow \infty} d\left(\underline{Z}_{n}, R+W \cap W^{\prime}\right)=0 .
$$

To see this we may write $\left\{f_{i}=0\right\}, i=1, \ldots, s$, and $\left\{g_{i}=0\right\}, i=1, \ldots, r$, the linear equations defining $W$ and $W^{\prime}$ respectively. Then $f_{i}\left(P_{n}\right)=0$ and $g_{i}\left(P_{n}\right) \rightarrow 0$. By extracting from the system $\left\{f_{i}=0, g_{i}=0\right\}$ a maximal free subsystem that we call $\left\{h_{i}\right\}, i=1, \ldots, t$, we obtain a system of equations defining $W \cap W^{\prime}$. By the above, $h_{i}\left(P_{n}\right) \rightarrow 0$. We can make a change of variables $\left(z_{1}, \ldots, z_{2 g}\right)$ (which does not affect the property of convergence of distances to zero) so that $z_{i}=h_{i}$ for $i \in\{1, \ldots, t\}$. This shows that $d\left(P_{n}, W \cap W^{\prime}\right) \rightarrow 0$ and therefore $d\left(\underline{Z}_{n}, R+W \cap W^{\prime}\right) \rightarrow 0$.

As the property $d\left(\underline{Z}_{n}, R+W \cap W^{\prime}\right) \rightarrow 0$ is true for all sequences $\left(\underline{Z}_{n}\right)$ of points of $C_{\alpha}$ tending to the point at infinity, we get that $R+W \cap W^{\prime}$ is asymptotic to $C_{\alpha}$.

Let $P_{\alpha}+W_{\alpha}$ be the smallest real weakly pre-special subvariety of $\mathbb{C}^{g}$ which is asymptotic to $C_{\alpha}$. Let $\mathbb{T}_{\alpha}=W_{\alpha} / W_{\alpha} \cap \Gamma$ and $\mathbb{T}_{\alpha}^{\prime}=\pi\left(P_{\alpha}\right)+\mathbb{T}_{\alpha}$. We say that $\mathbb{T}_{\alpha}^{\prime}$ is the asymptotic Mumford-Tate torus of $C_{\alpha}$. By definition, for all $\alpha$ we have the inclusion $\mathbb{T}_{\alpha}^{\prime} \subset \mathbb{T}_{C}^{\prime}$. We remark that even if the infinite branches of $C$ depend on the choice of some small enough neighbourhood of some point at infinity of the curve, the associated asymptotic Mumford-Tate tori does not depend of this choice.

Lemma 2.2. We have

$$
\left.\overline{\pi\left(C_{\alpha}\right.}\right)-\pi\left(C_{\alpha}\right) \subset \mathbb{T}_{\alpha}^{\prime}
$$

Proof. Let $P_{\infty}$ be a point of $\overline{\pi\left(C_{\alpha}\right)}-\pi\left(C_{\alpha}\right)$. There exists a sequence $Q_{n}$ of points of $C_{\alpha}$ tending to the point at infinity of $C_{\alpha}$ such that $P_{n}:=\pi\left(Q_{n}\right)$ tends to $P_{\infty}$. By hypothesis, $d\left(Q_{n}, P_{\alpha}+W_{\alpha}\right)$ tends to 0 as $n$ tends to $\infty$. Therefore $d\left(P_{n}, \mathbb{T}_{\alpha}^{\prime}\right)$ tends to 0 . As $\mathbb{T}_{\alpha}^{\prime}$ is a closed subset of $A$, we find that $P_{\infty} \in \mathbb{T}_{\alpha}^{\prime}$. 
The following proposition summarizes what we have obtained so far.

Proposition 2.3. Let $C$ be a curve in $\mathbb{C}^{g}$. Let $C_{1}, \ldots, C_{r}$ be the set of all branches of $C$ through all points at infinity. Let $\mathbb{T}_{1}^{\prime}, \ldots, \mathbb{T}_{r}^{\prime}$ be the associated asymptotic Mumford-Tate tori. Then

$$
\overline{\pi(C)} \subset \pi(C) \cup \bigcup_{\alpha=1}^{r} \mathbb{T}_{\alpha}^{\prime} \subset \mathbb{T}_{C}^{\prime} .
$$

The main result of the paper is the following theorem.

Theorem 2.4. Let $C$ be a curve in $\mathbb{C}^{g}$. Let $C_{1}, \ldots, C_{r}$ be the set of all branches of $C$ through all points at infinity. Let $\mathbb{T}_{1}^{\prime}, \ldots, \mathbb{T}_{r}^{\prime}$ be the associated asymptotic Mumford-Tate tori.

(i) We have

$$
\overline{\pi(C)}=\pi(C) \cup \bigcup_{\alpha=1}^{r} \mathbb{T}_{\alpha}^{\prime} .
$$

(ii) Let $\mu_{\alpha}$ be the canonical probability measure on $\mathbb{T}_{\alpha}^{\prime}$. There exists positive real numbers $c_{1}, \ldots, c_{r}$ such that $\mu_{C, R}$ converges weakly to $\sum_{\alpha=1}^{r} c_{\alpha} \mu_{\alpha}$.

The first part of the theorem is a consequence of Proposition 2.3 and the second part of the theorem. In the rest of the paper we will give a proof of the second part of the theorem using some tools from harmonic analysis. Note also that very often at least one of the asymptotic Mumford-Tate tori of $C$ is just the Mumford-Tate torus of $C$. In this case, we have $\overline{\pi(C)}=\mathbb{T}_{C}^{\prime}$. General examples of this type will be given in Section 6 .

\section{Weyl criterion and Mumford-Tate tori}

3.1. Weyl criterion. Let $\langle\cdot, \cdot\rangle$ be the canonical Hermitian scalar product on $\mathbb{C}^{g}$. For any vectors $z, z^{\prime}$ of $\mathbb{C}^{g}$, we denote by $\left(z, z^{\prime}\right):=\operatorname{Re}\left(\left\langle z, z^{\prime}\right\rangle\right)$ the real part of $\left\langle z, z^{\prime}\right\rangle$. Then $(\cdot, \cdot)$ is the usual Euclidean product on $\mathbb{C}^{g} \simeq \mathbb{R}^{2 g}$. For any subset $E$ of $\mathbb{R}^{2 g}$ we write $E^{\perp}$ for the orthogonal complement of $E$ with respect to the Euclidean product $(\cdot, \cdot)$.

For any $\theta \in \mathbb{C}^{g}$, we denote by $\chi_{\theta}$ the function on $\mathbb{C}^{g}$ such that $\chi_{\theta}(z)=\exp (2 \pi i(\theta, z))$.

Let $\widehat{\Gamma}$ be the dual lattice of $\Gamma$ defined as

$$
\widehat{\Gamma}=\left\{\theta \in \mathbb{C}^{g}:(\theta, \gamma) \in \mathbb{Z} \text { for all } \gamma \in \Gamma\right\} .
$$

The group of characters $X^{*}(A)$ of the abelian variety $A$ viewed as a real torus of dimension $2 g$ is a free $\mathbb{Z}$-module of rank $2 g$. We denote by $\mathbf{1}_{A}$ the trivial character of $A$. We have an isomorphism

$$
\widehat{\Gamma} \simeq X^{*}(A)
$$

given by $\theta \mapsto \chi_{\theta}$. The Weyl criterion says that a sequence of probability measures $\mu_{n}$ on $A$ converges weakly to a probability measure $\mu$ if and only if for all $\theta \in \widehat{\Gamma}$ we have

$$
\mu_{n}\left(\chi_{\theta}\right) \rightarrow \mu\left(\chi_{\theta}\right) \text { as } n \rightarrow \infty .
$$

Let $\mathbb{T}=W_{\mathbb{T}} /\left(\Gamma \cap W_{\mathbb{T}}\right)$ be a real subtorus of $A$. Let $X^{*}(\mathbb{T})$ be the set of characters of $\mathbb{T}$. We denote by $\mathbf{1}_{\mathbb{T}}$ the trivial character of $\mathbb{T}$. Let $\theta \in \widehat{\Gamma}$. The restriction $\left(\chi_{\theta}\right)_{\mid \mathbb{T}}$ of $\chi_{\theta}$ to $\mathbb{T}$ 
is a character of $\mathbb{T}$. Let $N(\mathbb{T})$ be the free $\mathbb{Z}$-submodule of $X^{*}(A)$ defined by

$$
N(\mathbb{T}):=\left\{\chi_{\theta} \in X^{*}(A):\left(\chi_{\theta}\right)_{\mid \mathbb{T}}=\mathbf{1}_{\mathbb{T}}\right\} .
$$

We have an exact sequence

$$
0 \rightarrow N(\mathbb{T}) \rightarrow X^{*}(A) \rightarrow X^{*}(\mathbb{T}) \rightarrow 0 .
$$

If we identify $\widehat{\Gamma}$ and $X^{*}(A)$, we can realize $N(\mathbb{T})$ as a subset of $\mathbb{C}^{g} \simeq \mathbb{R}^{2 g}$. Then $N(\mathbb{T})$ is a lattice in

$$
N(\mathbb{T}) \otimes \mathbb{R} \simeq W_{\mathbb{T}}^{\perp} \subset \mathbb{R}^{2 g}
$$

and $N(\mathbb{T})=\widehat{\Gamma} \cap(N(\mathbb{T}) \otimes \mathbb{R})$. Let $\mathbb{T}^{\vee}$ be the real torus $A / \mathbb{T}$. Then $\mathbb{T}^{\vee} \simeq N(\mathbb{T}) \otimes \mathbb{R} / N(\mathbb{T})$ and we have an identification $N(\mathbb{T})=X^{*}\left(\mathbb{T}^{\vee}\right)$.

Let $P$ be a point of $\mathbb{C}^{g}$. Let $\mathbb{T}^{\prime}=\pi(P)+\mathbb{T}$. Let $\mu_{\mathbb{T}^{\prime}}$ be the canonical probability measure on $\mathbb{T}^{\prime}$. Then for all $\theta$ in $\widehat{\Gamma}$, we have

$$
\int_{A} \chi_{\theta} d \mu_{\mathbb{T}^{\prime}}=\int_{A} \exp (2 \pi i(\theta, z)) d \mu_{\mathbb{T}^{\prime}}=\exp (2 i \pi(P, \theta)) \quad \text { if } \theta \in N(\mathbb{T})
$$

and

$$
\int_{A} \chi_{\theta} d \mu_{\mathbb{T}^{\prime}}=\int_{A} \exp (2 \pi i(\theta, z)) d \mu_{\mathbb{T}^{\prime}}=0 \quad \text { if } \theta \notin N(\mathbb{T}) .
$$

As the proof of Theorem 2.4 is an application of the Weyl criterion, we will need to understand the group of characters of the Mumford-Tate torus of an algebraic subvariety of $\mathbb{C}^{g}$ and of the asymptotic Mumford-Tate tori of a branch of a curve in $\mathbb{C}^{g}$. The relevant results are given in the next two subsections.

3.2. Characters of the Mumford-Tate torus. Let $\Theta$ be an irreducible subvariety of $\mathbb{C}^{g}$. Let $P$ be a point of $\Theta$. Let

$$
W_{\Theta}=\mathrm{MT}(\Theta-P) \otimes \mathbb{R}
$$

and $\mathbb{T}_{\Theta}=W_{\Theta} /\left(\Gamma \cap W_{\Theta}\right)$. The Mumford-Tate torus of $\Theta$ is $\mathbb{T}_{\Theta}^{\prime}=\pi(P)+\mathbb{T}_{\Theta}$. By the discussion of the previous subsection we have

$$
N\left(\mathbb{T}_{\Theta}\right)=X^{*}\left(A / \mathbb{T}_{\Theta}\right)=W_{\Theta}^{\perp} \cap \widehat{\Gamma} .
$$

We will need the following lemma.

Lemma 3.1. We have

$$
N\left(\mathbb{T}_{\Theta}\right)=\left\{\chi_{\theta}, \theta \in \widehat{\Gamma} \cap(\Theta-P)^{\perp}\right\} \simeq \widehat{\Gamma} \cap(\Theta-P)^{\perp} .
$$

Proof. Let $N^{*}\left(\mathbb{T}_{\Theta}\right)=\widehat{\Gamma} \cap(\Theta-P)^{\perp}$. As $\Theta-P \subset W_{\Theta}$, we get

$$
N\left(\mathbb{T}_{\Theta}\right) \subset N^{*}\left(\mathbb{T}_{\Theta}\right) .
$$

Moreover, $N^{*}\left(\mathbb{T}_{\Theta}\right)$ is a saturated $\mathbb{Z}$-submodule of $\widehat{\Gamma}$ in the sense that if there exists $\theta \in \widehat{\Gamma}$ and a non-zero integer $n$ such that $n \theta \in N^{*}\left(\mathbb{T}_{\Theta}\right)$ then $\theta \in N^{*}\left(\mathbb{T}_{\Theta}\right)$. As a consequence there is a real subtorus $\mathbb{T}_{\Theta}^{*}$ of $A$ and an exact sequence

$$
0 \rightarrow N^{*}\left(\mathbb{T}_{\Theta}\right) \rightarrow \widehat{\Gamma} \simeq X^{*}(A) \rightarrow X^{*}\left(\mathbb{T}_{\Theta}^{*}\right) \rightarrow 0 .
$$

By definition, $\pi(\Theta-P) \subset \mathbb{T}_{\Theta}^{*}$ and as $N\left(\mathbb{T}_{\Theta}\right) \subset N^{*}\left(\mathbb{T}_{\Theta}\right)$, we have $\mathbb{T}_{\Theta}^{*} \subset \mathbb{T}_{\Theta}$. By the definition of the Mumford-Tate tori of $\Theta-P$, we find that $\mathbb{T}_{\Theta}^{*}=\mathbb{T}_{\Theta}$ and $N^{*}\left(\mathbb{T}_{\Theta}\right)=N\left(\mathbb{T}_{\Theta}\right)$. 
3.3. Characters of the asymptotic Mumford-Tate Tori of the branches of a curve. Let $C$ be an irreducible algebraic curve in $\mathbb{C}^{g}$, and let $C_{\alpha}$ be an infinite branch of $C$. Let $P_{\alpha}+W_{\alpha}$ be the smallest real weakly pre-special subvariety which is asymptotic to $C_{\alpha}$, and let $\mathbb{T}_{\alpha}^{\prime}=\pi\left(P_{\alpha}\right)+\mathbb{T}_{\alpha}$ be the associated asymptotic torus.

Let $\underline{Z}=\left(z_{1}, \ldots, z_{g}\right)$ be a system of coordinates on $C_{\alpha}$. We fix a coordinate $z=z_{i}$ which is unbounded as $\underline{Z}$ varies in $C_{\alpha}$. For all $j \in\{1, \ldots, g\}$ we have a Puiseux expansion of the form

$$
z_{j}=\sum_{n \geq 0} a_{n, j} z^{\alpha_{j}-\frac{n}{e_{j}}}
$$

for some $\alpha_{j} \in \mathbb{Q}$ and some $e_{j} \in \mathbb{N}^{*}$ (see [9, Chapter 4]). The sequence of complex numbers $a_{n, j}$ is such that the power series $\sum_{n \geq 0} a_{n, j} X^{n}$ has a radius of convergence $\rho_{j}>0$.

Let $a_{0}\left(z_{j}\right)$ be the constant term of $z_{j}$ in this expansion. Note that $a_{0}\left(z_{j}\right)=0$ if and only if $\alpha_{j}-\frac{n}{e_{j}} \neq 0$ for all $n \in \mathbb{N}$, or if there exists $n_{0} \in \mathbb{N}$ such that $\alpha_{j}-\frac{n_{0}}{e_{j}}=0$ and $a_{n_{0}, j}=0$. Let $Q_{\alpha}$ be the point of $\mathbb{C}^{g}$ such that

$$
Q_{\alpha}=\left(a_{0}\left(z_{1}\right), \ldots, a_{0}\left(z_{g}\right)\right) .
$$

Let $N_{\alpha}$ be the $\mathbb{Z}$-submodule of $\widehat{\Gamma}$ consisting of the $\theta=\left(\theta_{1}, \ldots, \theta_{g}\right) \in \widehat{\Gamma}$ such that the function

$$
\psi_{\theta}(\underline{Z})=(\underline{Z}, \theta)=\operatorname{Re}\left(\sum_{k=1}^{g} \overline{\theta_{k}} z_{k}\right)
$$

is bounded on $C_{\alpha}$. Then $N_{\alpha}$ is a saturated submodule of $\widehat{\Gamma}$. There exists therefore a real subtorus $\mathbb{S}_{\alpha}$ of $A$ and an exact sequence

$$
0 \rightarrow N_{\alpha} \rightarrow \widehat{\Gamma} \simeq X^{*}(A) \rightarrow X^{*}\left(\mathbb{S}_{\alpha}\right) \rightarrow 0
$$

Lemma 3.2. We have $\mathbb{T}_{\alpha}=\mathbb{S}_{\alpha}, N\left(\mathbb{T}_{\alpha}\right)=N_{\alpha}$ and

$$
\mathbb{T}_{\alpha}^{\prime}=\pi\left(Q_{\alpha}\right)+\mathbb{S}_{\alpha}=\pi\left(P_{\alpha}\right)+\mathbb{S}_{\alpha}
$$

Proof. Let $\theta=\left(\theta_{1}, \ldots, \theta_{g}\right) \in \widehat{\Gamma}$. Let $H_{\theta}$ be the real hyperplane of $\mathbb{C}^{g} \simeq \mathbb{R}^{2 g}$ with equation

$$
\operatorname{Re}\left(\sum_{k=1}^{g} \overline{\theta_{k}} z_{k}\right)=0 .
$$

Then $\Gamma_{\theta}:=\Gamma \cap H_{\theta}$ is a lattice in $H_{\theta}$.

If there exists a point $Q$ such that $C_{\alpha}$ is asymptotic $Q+H_{\theta}$, then the distance $d\left(\underline{Z}, H_{\theta}\right)$ is bounded as $\underline{Z}$ varies in $C_{\alpha}$. By the computation of the distance of a point to a hyperplane this implies that the function $\operatorname{Re}\left(\sum_{k=1}^{g} \bar{\theta}_{k} z_{k}\right)$ is bounded when $\underline{Z}$ varies in $C_{\alpha}$.

If $\operatorname{Re}\left(\sum_{k=1}^{g} \overline{\theta_{k}} z_{k}\right)$ is bounded when $\underline{Z}$ varies in $C_{\alpha}$, the coefficients of the positive powers of $z$ in the Puiseux expansion of

$$
\psi_{\theta}(\underline{Z})=\operatorname{Re}\left(\sum_{k=1}^{g} \overline{\theta_{k}} z_{k}\right)
$$

are vanishing. Moreover, the function $\psi_{\theta}\left(\underline{Z}-Q_{\alpha}\right)$ tends to zero as $z$ tends to $\infty$. As a consequence, $C_{\alpha}$ is asymptotic to $Q_{\alpha}+H_{\theta}$ and

$$
P_{\alpha}+W_{\alpha} \subset Q_{\alpha}+H_{\theta}=P_{\alpha}+H_{\theta} .
$$


If $W_{\alpha} \neq \mathbb{C}^{g}$, then $W_{\alpha}$ is the intersection of the hyperplanes $H_{\theta}$ with $\theta \in \widehat{\Gamma}$ such that $C_{\alpha}$ is asymptotic to $P_{\alpha}+H_{\theta}$. By the previous discussion, we have

$$
W_{\alpha}=\bigcap_{\theta \in N_{\alpha}} H_{\theta} .
$$

This shows that

$$
\mathbb{T}_{\alpha}=\mathbb{S}_{\alpha}=W_{\alpha} /\left(\Gamma \cap W_{\alpha}\right)
$$

and that

$$
\mathbb{T}_{\alpha}^{\prime}=\pi\left(Q_{\alpha}\right)+\mathbb{S}_{\alpha}=\pi\left(P_{\alpha}\right)+\mathbb{S}_{\alpha} .
$$

If $W_{\alpha}=\mathbb{C}^{g}$, then there is no hyperplane $H_{\theta}$ such that $C_{\alpha}$ is asymptotic to $P_{\alpha}+H_{\theta}$ for some point $P_{\alpha}$. Therefore $N_{\alpha}$ is trivial and

$$
\mathbb{S}_{\alpha}=\mathbb{C}^{g}=\mathbb{T}_{\alpha}
$$

\section{Local computations for oscillatory integrals in the complex plane}

In this section we give the computations for oscillatory integrals which are relevant for the application of the Weyl criterion in the proof of Theorem 2.4.

We consider a function $\phi$ of a complex variable $z$ defined for $|z|>A$ for a suitable real number $A$. We assume moreover that we have an expansion of the form

$$
\phi(z)=\sum_{n \geq 0} a_{n} z^{\alpha-\frac{n}{e}},
$$

where $e$ is a positive integer, $0 \leq \alpha$ is a rational number and $\left(a_{n}\right)_{n \in \mathbb{N}}$ is a sequence of complex numbers such that the power series $\sum a_{n} T^{n}$ has a positive radius of convergence $\rho$. If $\alpha \neq 0$, we assume that $a_{0} \neq 0$. By making $A$ larger we may and do assume that $A>1$ and if $\rho \neq \infty$ that

$$
\frac{1}{A^{\frac{1}{e}}}<\frac{\rho}{2}
$$

For a complex number $z=r \exp (i \theta)$ with $\theta \in[0,2 \pi[$ and a positive integer $k$ we define

$$
z^{\frac{1}{k}}:=r^{\frac{1}{k}} \exp \left(\frac{i \theta}{k}\right)
$$

The purpose of this part is to study the behaviour for large $R$ of the integral

$$
J_{\phi}(R)=\int_{A<|z|<R} \exp (i \operatorname{Re}(\phi(z))) \frac{i d z \wedge d \bar{z}}{2 R^{2}} .
$$

Theorem 4.1. For any function $\phi$ satisfying the previous properties

(i) If $\alpha>0$, then

$$
J_{\phi}(R) \rightarrow 0
$$

as $R \rightarrow \infty$.

(ii) If $\alpha=0$, then

$$
J_{\phi}(R) \rightarrow \pi \exp \left(i \operatorname{Re}\left(a_{0}\right)\right)
$$

as $R \rightarrow \infty$. 
We write $z=r \exp (i \theta), a_{n}=A_{n} \exp \left(i \gamma_{n}\right)$. Then

$$
\psi(r, \theta):=\operatorname{Re}(\phi(z))=\sum_{n \geq 0} A_{n} r^{\alpha-\frac{n}{e}} b_{n}(\theta)
$$

with

$$
b_{n}(\theta):=\cos \left(\left(\alpha-\frac{n}{e}\right) \theta+\gamma_{n}\right) .
$$

With these notations we find that

$$
J_{\phi}(R)=\int_{0}^{2 \pi} \int_{\frac{A}{R}}^{1} \exp (i \psi(R t, \theta)) t d t d \theta .
$$

We fix $\theta \in[0,2 \pi]$. For $t \in\left[\frac{A}{R}, 1\right]$ we define

$$
f_{\theta, R}(t):=\psi(R t, \theta)=\sum_{n \geq 0} A_{n} R^{\alpha-\frac{n}{e}} t^{\alpha-\frac{n}{e}} b_{n}(\theta) .
$$

The main input in the proof of the first part of Theorem 4.1 is the Van der Corput lemma that can be stated in the following way.

Lemma 4.2 (Van der Corput lemma). Let $k \geq 2$ be an integer. There exists a constant $C=C_{k}$ such that for any real numbers $a<b$, any functions $f \in C^{k}([a, b])$ such that $\left|f^{(k)}(x)\right|>\lambda>0$ on $[a, b]$ and any function $\psi \in C^{1}([a, b])$,

$$
\left|\int_{a}^{b} \exp (i f(x)) \psi(x) d x\right| \leq C_{k}\left(\|\psi\|_{L^{\infty}([a, b])}+\left\|\psi^{\prime}\right\|_{L^{1}([a, b])}\right) \lambda^{-\frac{1}{k}} .
$$

If $k=1$ and $f^{\prime}$ is monotone, the conclusion also holds.

A proof of this result is given in [7]. The next lemma summarizes what we need in order to be able to apply the Van der Corput lemma.

Lemma 4.3. The following statements hold.

(i) We have

$$
f_{\theta, R}^{\prime}(t)=\sum_{n \geq 0}\left(\alpha-\frac{n}{e}\right) A_{n} R^{\alpha-\frac{n}{e}} t^{\alpha-1-\frac{n}{e}} b_{n}(\theta)
$$

and

$$
f_{\theta, R}^{\prime \prime}(t)=\sum_{n \geq 0}\left(\alpha-\frac{n}{e}\right)\left(\alpha-1-\frac{n}{e}\right) A_{n} R^{\alpha-\frac{n}{e}} t^{\alpha-2-\frac{n}{e}} b_{n}(\theta) .
$$

(ii) Assume that $\alpha=1$. Let $\eta$ be a positive real number such that $\eta<\frac{1}{2 e}$. There exists a positive constant $c_{1}=c_{1}(\eta)$ such that the following holds. For $R$ big enough, for all $\theta \in[0,2 \pi]$ such that $\left|A_{0} b_{0}(\theta)\right|>R^{-\eta}$ and for $t \in\left[\frac{A}{R^{1 / 2}}, 1\right]$ we have

$$
\left|f_{\theta, R}^{\prime}(t)\right|>c_{1} R^{1-\eta}
$$


(iii) Assume $\alpha>0$. If $\alpha=1$, assume that the smallest positive integer $n_{0}$ such that $A_{n_{0}} \neq 0$ verifies $n_{0}<e$. There exist real numbers $\beta>0, \frac{1}{2}>\delta>0$ and $\mu>0$ such that for all $t \in\left[\frac{A}{R^{\delta}}, 1\right]$ and all $\theta \in[0,2 \pi]$ such that

$$
R^{-\beta}< \begin{cases}\left|\alpha(\alpha-1) A_{0} b_{0}(\theta)\right| & \text { if } \alpha \neq 1, \\ \left|\frac{n_{0}}{e}\left(1-\frac{n_{0}}{e}\right) A_{n_{0}} b_{n_{0}}(\theta)\right| & \text { if } \alpha=1,\end{cases}
$$

we have

$$
\left|f_{\theta, R}^{\prime \prime}(t)\right|>c_{2} R^{\mu}
$$

for a constant $c_{2}>0$.

(iv) Assume $\alpha=0$ or $\alpha=1$ and for all $k>0$ such that $0<k<e$ we have $A_{k}=0$. Let $n_{0}$ be the smaller positive integer $k$ such that $a_{k} \neq 0$. There exist real numbers $\beta>0$ and $\delta>0$ such that for $t \in\left[\frac{A}{R^{\delta}}, 1\right]$ and all $\theta \in[0,2 \pi]$ such that $\left|A_{n_{0}} b_{n_{0}}(\theta)\right|>R^{-\beta}$ the function $f_{\theta, R}^{\prime \prime}(t)$ is non-vanishing.

Proof. To prove (i) we remark that for $t \in\left[\frac{A}{R}, 1\right]$ and $n$ big enough we have

$$
\left|\left(\alpha-\frac{n}{e}\right) A_{n} R^{\alpha-\frac{n}{e}} t^{\alpha-1-\frac{n}{e}} b_{n}(\theta)\right| \leq 2 n\left|A_{n}\right| R A^{\alpha-1}\left(\frac{1}{A^{\frac{1}{e}}}\right)^{n} .
$$

The series with general term

$$
B_{n}=2 n\left|A_{n}\right| R A^{\alpha-1}\left(\frac{1}{A^{\frac{1}{e}}}\right)^{n}
$$

is convergent as the power series $\sum A_{n} T^{n}$ has a convergence radius $\rho>0$ and as by hypothesis $\frac{1}{A^{1 / e}}<\frac{\rho}{2}$. This shows the validity of the expression for $f_{\theta, R}^{\prime}(t)$. The proof for $f_{\theta, R}^{\prime \prime}(t)$ is done in the same way.

To prove (ii) we assume that $\alpha=1$. We use for $t \in\left[\frac{A}{R^{1 / 2}}, 1\right]$ the upper bound

$$
\left|\left(1-\frac{n}{e}\right) A_{n} R^{1-\frac{n}{e}} t^{-\frac{n}{e}} b_{n}(\theta)\right| \leq(n+1)\left|A_{n}\right| R^{1-\frac{1}{2 e}}\left(\frac{1}{A^{\frac{1}{e}}}\right)^{n} \quad \text { for all } n>0 .
$$

Let $c_{n}:=(n+1)\left|A_{n}\right|$. The power series $\sum c_{n} T^{n}$ has a radius of convergence $\rho$ and is therefore bounded for $|T| \leq \frac{\rho}{2}$. By hypothesis, $\frac{1}{A^{1 / e}}<\frac{\rho}{2}$. Therefore if $R$ is large enough, as $\eta<\frac{1}{2 e}$, if $\left|A_{0} b_{0}(\theta)\right|>R^{-\eta}$ and $t \in\left[\frac{A}{R^{1 / 2}}, 1\right]$, we get

$$
\left|f_{\theta, R}^{\prime}(t)\right|>c_{1}(\eta) R^{1-\eta}
$$

For part (iii) we assume first that $0<\alpha \neq 1$. Let $\beta=\min \left(\frac{\alpha}{2}, \frac{1}{4 e}\right)$. Let $\delta>0$. If $\alpha>2$, assume that

$$
\delta(\alpha-2) \leq \frac{1}{4 e}<1
$$

Assume that

$$
\left|\alpha(\alpha-1) A_{0} b_{0}(\theta)\right|>R^{-\beta} .
$$

Then for all $t \in\left[\frac{A}{R^{\delta}}, 1\right]$ we have

$$
\left|\alpha(\alpha-1) R^{\alpha} A_{0} b_{0}(\theta) t^{\alpha-2}\right| \geq R^{\alpha-\beta} \quad \text { if } \alpha \leq 2
$$


and

$$
\left|\alpha(\alpha-1) R^{\alpha} A_{0} b_{0}(\theta) t^{\alpha-2}\right| \geq A^{-2} R^{\alpha-\beta-\delta(\alpha-2)} \quad \text { if } \alpha>2 .
$$

Let $\mu=\max \left(\frac{\alpha}{2}, \alpha-\frac{1}{2 e}\right)$. Remark that if $\alpha \geq 2$, then $\beta=\frac{1}{4 e}$ and $\mu=\alpha-\frac{1}{2 e}$. Using the upper bound (4.4) for $\delta$ when $\alpha>2$ shows that for all $\alpha>0$ with $\alpha \neq 1$ we have

$$
\left|\alpha(\alpha-1) R^{\alpha} A_{0} b_{0}(\theta) t^{\alpha-2}\right| \geq A^{-2} R^{\mu} .
$$

Assume moreover that $\delta>0$ satisfies

$$
\frac{1}{2 e}<\alpha \delta+\frac{1}{e}(1-\delta)-2 \delta
$$

and $\delta<\frac{1}{2}$. Remark that such a $\delta$ exists as the limit when $\delta$ tends to zero of the right-hand side of the equation is $\frac{1}{e}>\frac{1}{2 e}$.

For all $t \in\left[\frac{A}{R^{\delta}}, 1\right]$ and all $n \geq 1$ such that $\alpha-2-\frac{n}{e}<0$ we have

$$
\left|\left(\alpha-\frac{n}{e}\right)\left(\alpha-1-\frac{n}{e}\right) A_{n} R^{\alpha-\frac{n}{e}} t^{\alpha-2-\frac{n}{e}} b_{n}(\theta)\right| \leq R^{\lambda_{1}}(n+1)^{2}\left|A_{n}\right| A^{\alpha-2}\left(\frac{1}{A^{\frac{1}{e}}}\right)^{n} .
$$

with

$$
\lambda_{1}=\alpha(1-\delta)-\frac{1}{e}(1-\delta)+2 \delta<\mu
$$

by our choice of $\delta$.

For all $t \in\left[\frac{A}{R^{\delta}}, 1\right]$ and all $n \geq 1$ such that $\alpha-2-\frac{n}{e} \geq 0$ we have

$$
\left|\left(\alpha-\frac{n}{e}\right)\left(\alpha-1-\frac{n}{e}\right) A_{n} R^{\alpha-\frac{n}{e}} t^{\alpha-2-\frac{n}{e}} b_{n}(\theta)\right| \leq R^{\lambda_{2}}(n+1)^{2}\left|A_{n}\right|
$$

with $\lambda_{2}=\alpha-\frac{n}{e}<\mu$. Let $\lambda=\max \left(\lambda_{1}, \lambda_{2}\right)$. Then $\lambda<\mu$.

Let $d_{n}:=(n+1)^{2}\left|A_{n}\right| A^{\alpha-2}$. The power series $\sum d_{n} T^{n}$ has a radius of convergence $\rho$ and is therefore bounded for $|T| \leq \frac{\rho}{2}$. By hypothesis, $\frac{1}{A^{1 / e}}<\frac{\rho}{2}$. Therefore there exists a positive constant $c$ such that for all $R$ big enough,

$$
\left|\sum_{n \geq 1}\left(\alpha-\frac{n}{e}\right)\left(\alpha-1-\frac{n}{e}\right) A_{n} R^{\alpha-\frac{n}{e}} t^{\alpha-2-\frac{n}{e}} b_{n}(\theta)\right| \leq c R^{\lambda} .
$$

In view of equation (4.7) and of the expression for $f_{\theta, R}^{\prime \prime}(t)$ obtained in part (i) of the lemma this finishes the proof of part (iii) when $\alpha \neq 1$.

The proof of part (iii) when $\alpha=1$ is obtained by considering the function

$$
g_{\theta, R}(t)=f_{\theta, R}(t)-A_{0} R t b_{0}(\theta) .
$$

We have $g_{\theta, R}^{\prime \prime}(t)=f_{\theta, R}^{\prime \prime}(t)$ and $g_{\theta, R}(t)$ has an expansion of the form

$$
g_{\theta, R}(t)=\sum_{n \geq 0} A_{n+n_{0}} R^{\alpha_{1}-\frac{n}{e}} t^{\alpha_{1}-\frac{n}{e}} b_{n+n_{0}}(\theta)
$$

with $\alpha_{1}=1-\frac{n_{0}}{e}>0$ and $A_{n_{0}} \neq 0$. Therefore $g_{\theta, R}(t)$ satisfies the hypothesis of part (iii) with $\alpha_{1} \neq 1$. This finishes the proof of part (iii) of the lemma.

Part (iv) of the lemma is proven similarly. We may assume as previously that $\alpha=0$ by considering the function

$$
g_{\theta, R}(t)=f_{\theta, R}(t)-A_{0} R t b_{0}(\theta) .
$$


In the case $\alpha=0$ we choose $\beta=\frac{1}{2 e}$. Assume that

$$
\left|\frac{n_{0}}{e}\left(\frac{n_{0}}{e}+1\right) A_{n_{0}} b_{n_{0}}(\theta)\right|>R^{-\beta}
$$

Then for all $t \in\left[\frac{A}{R}, 1\right]$ we have

$$
\left|\frac{n_{0}}{e}\left(\frac{n_{0}}{e}+1\right) A_{n_{0}} b_{n_{0}}(\theta) R^{-\frac{n_{0}}{e}} t^{-2-\frac{n_{0}}{e}}\right| \geq R^{-\frac{n_{0}}{e}-\beta}=R^{-\frac{n_{0}+\frac{1}{2}}{e}} .
$$
have

Let $\delta>0$ such that $\left(\frac{n_{0}+1}{e}+2\right) \delta<\beta=\frac{1}{2 e}$. For all $t \in\left[\frac{A}{R^{\delta}}, 1\right]$ and all $n \geq n_{0}+1$ we

$$
\left|\frac{n}{e}\left(1+\frac{n}{e}\right) A_{n} R^{-\frac{n}{e}} t^{-2-\frac{n}{e}} b_{n}(\theta)\right| \leq R^{\lambda}(n+1)^{2}\left|A_{n}\right| A^{-2}\left(\frac{1}{A^{\frac{1}{e}}}\right)^{n} .
$$

with

$$
\lambda=-\frac{n_{0}+1}{e}(1-\delta)+2 \delta<-\frac{n_{0}+1}{e}+\beta=-\frac{n_{0}+\frac{1}{2}}{e}
$$

by our choice of $\delta$.

Let $d_{n}:=(n+1)^{2}\left|A_{n}\right| A^{-2}$. The power series $\sum d_{n} T^{n}$ has a radius of convergence $\rho$ and is therefore bounded for $|T| \leq \frac{\rho}{2}$. By hypothesis, $\frac{1}{A^{1 / e}}<\frac{\rho}{2}$. Therefore there exists a positive constant $c$ such that for all $R$ big enough,

$$
\left|\sum_{n \geq n_{0}+1} \frac{n}{e}\left(1+\frac{n}{e}\right) A_{n} R^{-\frac{n}{e}} t^{-2-\frac{n}{e}} b_{n}(\theta)\right| \leq c R^{\lambda} .
$$

This shows that

$$
f_{\theta, R}^{\prime \prime}(t)=\sum_{n \geq n_{0}} \frac{n}{e}\left(1+\frac{n}{e}\right) A_{n} R^{-\frac{n}{e}} t^{-2-\frac{n}{e}} b_{n}(\theta)
$$

is non-vanishing if $t \in\left[\frac{A}{R^{\delta}}, 1\right]$. This finishes the proof of the lemma.

We first prove the first part of Theorem 4.1. Let $\beta$ be the constant defined in part (iii) of the lemma if $\alpha \neq 1$ and let $\beta$ be the constant defined in part (iii) (resp. part (iv)) if $\alpha=1$ and the hypothesis of part (iii) (resp. part (iv)) holds.

If $\alpha \neq 1$, let $E_{1}$ be the subset of $\theta \in[0,2 \pi$ [ such that

$$
\left|\alpha(\alpha-1) A_{0} b_{0}(\theta)\right|>R^{-\beta} .
$$

If $\alpha=1$, let $n_{0}$ be the integer defined in part (iii) or in part (iv) of the lemma. Let $E_{1}$ be the subset of $\theta \in\left[0,2 \pi\left[\right.\right.$ such that $\left|A_{0} b_{0}(\theta)\right|>R^{-\beta}$ and such that

$$
\left|\frac{n_{0}}{e}\left(1-\frac{n_{0}}{e}\right) A_{n_{0}} b_{n_{0}}(\theta)\right|>R^{-\beta}
$$

if the hypothesis of part (iii) holds and such that

$$
\left|A_{n_{0}} b_{n_{0}}(\theta)\right|>R^{-\beta}
$$

if the hypothesis of part (iv) holds. 
Let $E_{2}$ such that $\left[0,2 \pi\right.$ [ is a disjoint union of $E_{1}$ and $E_{2}$. Then there exists $\epsilon_{1}>0$ such that $E_{2}$ is of Lebesgue measure bounded by $R^{-\epsilon_{1}}$ for $R$ big enough. We have

$$
J_{\phi}(R)=J_{\phi}^{1}(R)+J_{\phi}^{2}(R)
$$

with

$$
J_{\phi}^{1}(R)=\int_{\theta \in E_{1}} \int_{\frac{A}{R}}^{1} \exp (i(\psi(R t, \theta)) t d t d \theta
$$

and

$$
J_{\phi}^{2}(R)=\int_{\theta \in E_{2}} \int_{\frac{A}{R}}^{1} \exp (i(\psi(R t, \theta)) t d t d \theta .
$$

Then for all $R$ big enough $\left|J_{\phi}^{2}(R)\right| \leq R^{-\epsilon_{1}}$ by the previous discussion.

Let $\delta$ be the constant defined in part (iii) if $\alpha \neq 1$ or if the hypothesis of part (iii) holds. If $\alpha=1$ and the hypothesis of part (iv) holds, let $\delta$ be the constant defined in part (iv).

We then write

$$
J_{\phi}^{1}(R)=J_{\phi}^{3}(R)+J_{\phi}^{4}(R)
$$

with

$$
J_{\phi}^{3}(R)=\int_{\theta \in E_{1}} \int_{\frac{A}{R}}^{\frac{A}{R^{\delta}}} \exp (i(\psi(R t, \theta)) t d t d \theta
$$

and

$$
J_{\phi}^{4}(R)=\int_{\theta \in E_{1}} \int_{\frac{A}{R^{\delta}}}^{1} \exp (i(\psi(R t, \theta)) t d t d \theta
$$

Then

$$
\left|J_{\phi, \gamma}^{3}(R)\right|<2 \pi \frac{A^{2}}{R^{2 \delta}} .
$$

If $\alpha \neq 1$ or $\alpha=1$ and the hypothesis of part (iii) holds, then using the result of part (iii) of the lemma and the Van der Corput lemma with $k=2$ and $\lambda=c_{2} R^{\mu}$ we find that for all $R$ big enough

$$
\left|J_{\phi}^{4}(R)\right| \ll R^{-\frac{\mu}{2}}
$$

Assume finally that $\alpha=1$ and the hypothesis of part (iv) hold. Using the result of part (iv), of part (ii) and the Van der Corput lemma with $k=1$ and dividing into $t>\frac{A}{R^{1 / 2}}$ and $t \leq \frac{A}{R^{1 / 2}}$ in the computation of $J_{\phi}^{4}(R)$, shows that there exists $\epsilon_{2}>0$ such that

$$
\left|J_{\phi}^{4}(R)\right| \ll R^{-\epsilon_{2}}
$$

This finishes the proof to the first part of Theorem 4.1

For the proof of the second part of Theorem 4.1, we assume $\alpha=0$. Therefore we have an expansion of $\phi(z)$ of the form

$$
\phi(z)=a_{0}+\sum_{n \geq 1} a_{n} z^{-\frac{n}{e}} .
$$

As previously, we write

$$
f_{\theta, R}(t)=\sum_{n \geq 0} A_{n} R^{-\frac{n}{e}} t^{-\frac{n}{e}} b_{n}(\theta)
$$

and

$$
J_{\phi}(R)=\int_{0}^{2 \pi} \int_{\frac{A}{R}}^{1} \exp \left(i f_{\theta, R}(t)\right) t d t d \theta
$$


Lemma 4.4. There exists a positive constant $c$ such that

$$
\left|\sum_{n \geq 1} A_{n} R^{-\frac{n}{e}} t^{-\frac{n}{e}} b_{n}(\theta)\right| \leq c R^{-\frac{1}{2 e}}
$$

for all $t \in\left[\frac{A}{R^{1 / 2}}, 1\right]$ and all $\theta \in[0,2 \pi]$.

Proof. For all $n \geq 1$ and all $t \in\left[\frac{A}{R^{1 / 2}}, 1\right]$ we have

$$
\left|A_{n} R^{-\frac{n}{e}} t^{-\frac{n}{e}} b_{n}(\theta)\right| \leq R^{-\frac{1}{2 e}}\left|A_{n}\right|\left(\frac{1}{A^{1 / e}}\right)^{n} .
$$

This finishes the proof as the power series $\sum A_{n} T^{n}$ has radius $\rho>0$ and $\frac{1}{A^{1 / e}} \leq \frac{\rho}{2}$.

Lemma 4.5. There exists a positive constant $c^{\prime}$ such that

$$
\left|\exp \left(i \sum_{n \geq 1} A_{n} R^{-\frac{n}{e}} t^{-\frac{n}{e}} b_{n}(\theta)\right)-1\right| \leq c^{\prime} R^{-\frac{1}{2 e}}
$$

for all $t \in\left[\frac{A}{R^{1 / 2}}, 1\right]$ and all $\theta \in[0,2 \pi]$.

Proof. Let $\psi(t, R, \theta)=\sum_{n \geq 1} A_{n} R^{-\frac{n}{e}} t^{-\frac{n}{e}} b_{n}(\theta)$. We have

$$
|\exp (i \psi(t, R, \theta))-1|=\sqrt{(\cos (\psi(t, R, \theta))-1)^{2}+\sin (\psi(t, R, \theta))^{2}} .
$$

This finishes the proof using the inequality of Lemma 4.4 for $t \in\left[\frac{A}{R^{1 / 2}}, 1\right]$ and $\theta \in[0,2 \pi]$.

We can now give the proof of the second part of Theorem 4.1. Let

$$
J_{\phi}^{\prime}(R)=\int_{0}^{2 \pi} \int_{0}^{1} \exp \left(i f_{\theta, R}(t)\right) t d t d \theta .
$$

Then $J_{\phi}(R)-J_{\phi}^{\prime}(R) \rightarrow 0$ as $R \rightarrow \infty$. We just need to prove that

$$
J_{\phi}^{\prime}(R) \rightarrow \pi \exp \left(i \operatorname{Re}\left(a_{0}\right)\right)
$$

as $R \rightarrow \infty$. We have

$$
J_{\phi}^{\prime}(R)-\pi \exp \left(i \operatorname{Re}\left(a_{0}\right)\right)=\int_{0}^{2 \pi} \int_{0}^{1} \exp \left(i \operatorname{Re}\left(a_{0}\right)\right)\left(\exp \left(i f_{\theta, R}(t)\right)-1\right) t d t d \theta=J_{1}+J_{2}
$$

with

$$
J_{1}=\int_{0}^{2 \pi} \int_{0}^{\frac{A}{R^{1 / 2}}} \exp \left(i \operatorname{Re}\left(a_{0}\right)\right)\left(\exp \left(i f_{\theta, R}(t)\right)-1\right) t d t d \theta
$$

and

$$
J_{2}=\int_{0}^{2 \pi} \int_{\frac{A}{R^{1 / 2}}}^{1} \exp \left(i \operatorname{Re}\left(a_{0}\right)\right)\left(\exp \left(i f_{\theta, R}(t)\right)-1\right) t d t d \theta .
$$

Then $\left|J_{1}\right| \leq \frac{2 \pi A^{2}}{R}$ and $J_{2} \rightarrow 0$ as $R \rightarrow \infty$ by Lemma 4.5 . 


\section{Proof of Theorem 2.4}

Let $C$ be a curve in $\mathbb{C}^{g}$. Let $C_{1}, \ldots, C_{r}$ be the branches at infinity of $C$. We recall that we defined

$$
\omega:=\frac{i}{2} \sum_{k=1}^{g} d z_{k} \wedge d \bar{z}_{k}
$$

and the hypercube

$$
B(0, R)=\left\{\left(z_{1}, \ldots, z_{g}\right) \in \mathbb{C}^{g}:\left|z_{k}\right|<R \text { for all } k \in\{1, \ldots, g\}\right\} .
$$

Lemma 5.1. There exists a positive constant a such that

$$
\int_{C \cap B(0, R)} \frac{\omega}{R^{2}} \rightarrow a
$$

as $R \rightarrow \infty$.

Proof. The question only depends on the behaviour of $C$ near the points at infinity. We therefore just need to show that for each branch $C_{\beta}$ of $C$ there exists a positive constant $a_{\beta}$ such that

$$
\int_{C_{\beta} \cap B(0, R)} \frac{\omega}{R^{2}} \rightarrow a_{\beta}
$$

We fix a branch $C_{\beta}$ of $C$ and to simplify notations we write $D=C_{\beta}$. We just need to show that for all $j \in\{1, \ldots, g\}$ there exists a non-negative constant $a_{j}$ such that

$$
\psi_{j}(R):=\int_{D \cap B(0, R)} \frac{i d z_{j} \wedge d \bar{z}_{j}}{2 R^{2}} \rightarrow a_{j}
$$

and that there exists a $j \in\{1, \ldots, g\}$ such that $a_{j}>0$.

If $z_{j}$ is bounded when $\underline{Z}=\left(z_{1}, \ldots, z_{g}\right)$ varies on $D$, then $\psi_{j}(R) \rightarrow 0$. Let $j$ be such that $z_{j}$ is unbounded. Writing $z=z_{j}$ as in the previous section and using the theory of Puiseux expansions (see [9, Chapter 4]) we have

$$
z_{k}=\sum_{n \geq 0} a_{k, n} z^{\alpha_{k}-\frac{n}{e_{k}}}
$$

with $a_{k, 0} \neq 0, \alpha_{k} \in \mathbb{Q}$ and $e_{k} \in \mathbb{N}^{*}$.

If there exists a $k \in\{1, \ldots, g\}$ such that $\alpha_{k}>1=\alpha_{j}$, then for $|z|$ tending to infinity

$$
|z|=\left|\frac{z_{k}}{a_{k, 0}}\right|^{\frac{1}{\alpha_{k}}}(1+o(1)) .
$$

Then for $\underline{Z}=\left(z_{1}, \ldots, z_{g}\right) \in D \cap B(0, R)$, we have

$$
|z| \ll R^{\frac{1}{\alpha_{k}}}
$$

and

$$
\left|\psi_{j}(R)\right| \ll R^{\frac{2}{\alpha_{k}}-2} .
$$

In this case, $\psi_{j}(R) \rightarrow 0$. 
If $\alpha_{j}=\max _{k}\left(\alpha_{k}\right)$, we define

$$
E_{j}=\left\{k \in\{1, \ldots, g\}: \alpha_{k}=1=\alpha_{j}\right\} .
$$

When $\underline{Z}=\left(z_{1}, \ldots, z_{g}\right)$ varies in $D \cap B(0, R)$, the function $z=z_{j}$ varies in

$$
\left\{z \in \mathbb{C}: A_{j}<|z|<\frac{1}{\max _{k \in E_{j}}\left(\left|a_{k, 0}\right|\right)} R(1+o(1))\right\}
$$

for some positive constant $A_{j}$. In this situation there exists a positive constant $d_{j}$ such that

$$
\psi_{j}(R)=\frac{\pi d_{j}}{\max _{k \in E_{j}}\left(\left|a_{k, 0}\right|\right)}(1+o(1)) .
$$

Let $C_{\beta}$ be a branch of $C$ and let

$$
\mathbb{T}_{\beta}^{\prime}=\pi\left(P_{\beta}\right)+\mathbb{T}_{\beta}
$$

be the associated asymptotic Mumford-Tate torus. Let $\mu_{\beta}$ be the normalized Haar measure on $\mathbb{T}_{\beta}^{\prime}$. Let $\mu_{\beta_{R}}$ and $\mu_{\beta_{R}}^{\prime}$ be the measures on $A$ such that for all continuous $\Gamma$-invariant functions $f$ on $\mathbb{C}^{g}$,

$$
\mu_{\beta, R}(f)=\frac{\int_{C_{\beta} \cap B(0, R)} f \omega}{\int_{C_{\beta} \cap B(0, R)} \omega}
$$

and

$$
\mu_{\beta, R}^{\prime}(f)=\frac{\int_{C_{\beta} \cap B(0, R)} f \omega}{R^{2}} .
$$

Theorem 2.4 is now a consequence of the following proposition.

Proposition 5.2. The measures $\mu_{\beta, R}$ converge weakly to $\mu_{\beta}$ as $R \rightarrow \infty$. There is a positive constant $c_{\beta}$ such that $\mu_{\beta, R}^{\prime}$ converges weakly to $c_{\beta} \mu_{\beta}$ as $R \rightarrow \infty$.

Proof of Theorem 2.4. Let us first verify that the proposition implies Theorem 2.4. Let $f$ be a continuous $\Gamma$-invariant function on $\mathbb{C}^{g}$ and

$$
I_{f}(R)=\frac{\int_{C \cap B(0, R)} f \omega}{\int_{C \cap B(0, R)} \omega} .
$$

By Lemma 5.1,

$$
I_{f}(R)=\frac{\int_{C \cap B(0, R)} f \omega}{a R^{2}}+o(1)=\sum_{\beta=1}^{r} \int_{C_{\beta} \cap B(0, R)} \frac{f \omega}{a R^{2}}+o(1) .
$$

Using the proof of Lemma 5.1, we get the existence of positive constants $a_{\beta}$ for $\beta \in\{1, \ldots, r\}$ such that

$$
I_{f}(R)=\sum_{\beta=1}^{r} a_{\beta} \mu_{\beta, R}(f)+o(1) .
$$

Then by Proposition 5.2 we see that

$$
I_{f}(R) \rightarrow \sum_{\beta=1}^{r} a_{\beta} \mu_{\beta}(f) .
$$

This finishes the proof of Theorem 2.4 assuming Proposition 5.2. 
Proof of Proposition 5.2. By equation (5.1) in proof of Lemma 5.1, the two statements of Proposition 5.2 are equivalent.

Let $C_{\beta}$ be a branch of $C$. Let

$$
\mathbb{T}_{\beta}^{\prime}=\pi\left(P_{\beta}\right)+\mathbb{T}_{\beta}
$$

be the associated asymptotic torus. Let $N\left(\mathbb{T}_{\beta}\right)$ be as previously the subset of $\widehat{\Gamma} \simeq X^{*}(A)$ consisting of characters of $A$ which have a trivial restriction to $\mathbb{T}_{\beta}$. Let $\theta \in \widehat{\Gamma}$, we recall that

$$
\mu_{\beta}\left(\chi_{\theta}\right)=0 \quad \text { if } \theta \notin N\left(\mathbb{T}_{\beta}\right)
$$

and

$$
\mu_{\beta}\left(\chi_{\theta}\right)=\exp \left(2 i \pi\left(P_{\beta}, \theta\right)\right) \quad \text { if } \theta \in N\left(\mathbb{T}_{\beta}\right) .
$$

By Lemma 3.2, $N\left(\mathbb{T}_{\alpha}\right)$ is the subset of $\widehat{\Gamma}$ consisting of all $\theta=\left(\theta_{1}, \ldots, \theta_{g}\right)$ such that the function

$$
\psi_{\theta}(z):=\operatorname{Re}\left(\phi_{\theta}(z)\right)
$$

(where $\left.\phi_{\theta}(z)=\sum_{k=1}^{g} \overline{\theta_{k}} z_{k}\right)$ is bounded as $\underline{Z}=\left(z_{1}, \ldots, z_{g}\right)$ varies in $C_{\beta}$.

By the Weyl criterion we must check that there is a positive constant $c_{\beta}$ independent of $\theta$ such that

and

$$
\mu_{\beta, R}^{\prime}\left(\chi_{\theta}\right) \rightarrow 0 \quad \text { if } \theta \notin N\left(\mathbb{T}_{\beta}\right)
$$

$$
\mu_{\beta, R}^{\prime}\left(\chi_{\theta}\right) \rightarrow c_{\beta} \exp \left(2 i \pi\left(P_{\beta}, \theta\right)\right) \quad \text { if } \theta \in N\left(\mathbb{T}_{\beta}\right) .
$$

For $k \in\{1, \ldots, g\}$, let $\mu_{\beta, k, R}^{\prime}$ be the measure on $A$ such that for all continuous $\Gamma$-invariant function $f$ we have

$$
\mu_{\beta, k, R}^{\prime}(f)=\frac{\int_{C_{\beta} \cap B(0, R)} f i d z_{k} \wedge d \bar{z}_{k}}{2 R^{2}}
$$

By definition,

$$
\mu_{\beta, R}^{\prime}=\sum_{k=1}^{r} \mu_{\beta, k, R}^{\prime} .
$$

By the proof of Lemma 5.1, if $z_{j}$ is a coordinate which is bounded on $C_{\beta}$, then

$$
\mu_{\beta, j, R}^{\prime}\left(\chi_{\theta}\right) \rightarrow 0 \text { for all } \theta \in \widehat{\Gamma} \text {. }
$$

If $z=z_{j}$ is unbounded on $C_{\beta}$, we use the notations of the Puiseux expansions given in equation (5.2). Then, if there is a $k \in\{1, \ldots, g\}$ such that $\alpha_{k}>1=\alpha_{j}$, once more as in the proof of Lemma 5.1 we have

$$
\mu_{\beta, j, R}^{\prime}\left(\chi_{\theta}\right) \rightarrow 0 \quad \text { for all } \theta \in \widehat{\Gamma} .
$$

Let $z=z_{j}$ be unbounded on $C_{\beta}$ and assume that $\alpha_{j}=1=\max _{k}\left(\alpha_{k}\right)$. Then $\phi_{\theta}(z)$ admits a Puiseux expansion of the form considered in Section 4

$$
\phi_{\theta}(z)=\sum_{n \geq 0} a_{n} z^{\alpha-\frac{n}{e}}
$$

with $\alpha \leq 1$. Then $\phi_{\theta}(z)$ is unbounded if $\alpha>0$ and bounded if $\alpha \leq 0$. By the proof of Lemma 5.1 we have

$$
\mu_{\beta, j, R}^{\prime}\left(\chi_{\theta}\right)=\int_{A<|z|<c R} \exp \left(i \operatorname{Re}\left(\phi_{\theta}(z)\right) \frac{i d z \wedge d \bar{z}}{2 R^{2}}+o(1)\right.
$$

for some positive constants $A=A_{\beta, k}$ and $c=c_{\beta, k}$. 
By the first part of Theorem 4.1, if $\theta \notin N\left(\mathbb{T}_{\beta}\right)$, then

$$
\mu_{\beta, j, R}^{\prime}\left(\chi_{\theta}\right) \rightarrow 0 .
$$

Let $a_{0}\left(z_{k}\right)$ be the constant term in the Puiseux expansion of $z_{k}$ given by equation (5.2). Let $Q_{\beta}$ be the point of $\mathbb{C}^{g}$ with coordinates $Q_{\beta}=\left(a_{0}\left(z_{1}\right), \ldots, a_{0}\left(z_{g}\right)\right)$. Let $a_{0}\left(\psi_{\theta}\right)$ be the real part of the constant term in the Puiseux expansion of $\phi_{\theta}(z)$. Then

$$
a_{0}\left(\psi_{\theta}\right)=\operatorname{Re}\left(\sum_{i=1}^{g} \overline{\theta_{k}} a_{0}\left(z_{k}\right)\right)=\left(\theta, Q_{\beta}\right) .
$$

By the second part of Theorem 4.1, if $\theta \in N\left(\mathbb{T}_{\beta}\right)$, using equation (5.4) we obtain that there exists a positive constant $d_{\beta, j}$ such that

$$
\mu_{\beta, j, R}^{\prime}\left(\chi_{\theta}\right) \rightarrow d_{\beta, j} \exp \left(i a_{0}\left(\psi_{\theta}\right)\right)=d_{\beta, j} \exp \left(i\left(Q_{\beta}, \theta\right)\right) .
$$

By Lemma 3.2, we have

$$
\left(Q_{\beta}, \theta\right)=\left(P_{\beta}, \theta\right) .
$$

This finishes the proof of Proposition 5.2 and of Theorem 2.4.

\section{Examples}

We give in this part three examples illustrating the results of this paper.

6.1. Complex geodesic flows on abelian varieties. As in the previous section, $A$ is a complex abelian variety of dimension $g$. We fix a lattice $\Gamma$ such that $A \simeq \mathbb{C}^{g} / \Gamma$ and we denote by $\pi: \mathbb{C}^{g} \rightarrow A$ the uniformizing map. Conjectures 1.2 and 1.3 for $\Theta$ a complex linear subspace of $\mathbb{C}^{g}$ are simple applications of Weyl's criterion. In this case, $\Theta$ is a totally geodesic subvariety of $\mathbb{C}^{g}$.

Let $V$ be a $\mathbb{C}$-vector space of dimension 2 and let $\left(e_{1}, e_{2}\right)$ be a $\mathbb{C}$-basis of $V$. Let $\Gamma$ be the lattice

$$
\Gamma:=\mathbb{Z} e_{1} \oplus \mathbb{Z} \sqrt{-1} e_{1} \oplus \mathbb{Z} e_{2} \oplus \mathbb{Z} \sqrt{-5} e_{2} .
$$

Then $A:=A / \Gamma$ is an abelian variety of dimension 2 . In fact, $A$ is a product of two elliptic curves. Let $W$ be a sub-vector space $\mathbb{C}\left(e_{1}+e_{2}\right)$ of $V$. Then the Mumford-Tate group of $W$ is

$$
\operatorname{MT}(W)=\mathbb{Q}\left(e_{1}+e_{2}\right)+\mathbb{Q} \sqrt{-1} e_{1}+\mathbb{Q} \sqrt{-5} e_{2}
$$

and

$$
\mathrm{MT}(W) \otimes \mathbb{R}=\mathbb{R}\left(e_{1}+e_{2}\right)+\mathbb{R} \sqrt{-1} e_{1}+\mathbb{R} \sqrt{-5} e_{2} .
$$

As a consequence, $\operatorname{MT}(W) \otimes \mathbb{R} / \Gamma \cap \operatorname{MT}(W) \otimes \mathbb{R}$ is a real torus of real dimension 3. This shows that we can not expect that in Conjecture 1.2 the analytic closure of $\pi(\Theta)$ has a complex structure.

6.2. The curve $Z_{1} Z_{2}=1$. Let $A$ be a complex abelian surface and let

$$
\pi: \mathbb{C}^{2} \rightarrow A=\mathbb{C}^{2} / \Gamma
$$

be the uniformizing map. Let $C$ be the curve in $\mathbb{C}^{2}$ with equation

$$
Z_{1} Z_{2}=1 \text {. }
$$


There are two branches

$$
C_{1}=\left\{\left(Z_{1}, \frac{1}{Z_{1}}\right):\left|Z_{1}\right|>1\right\}
$$

and

$$
C_{2}=\left\{\left(\frac{1}{Z_{2}}, Z_{2}\right):\left|Z_{2}\right|>1\right\} .
$$

Let $\mathbb{T}_{1}^{\prime}=\pi\left(P_{1}\right)+\mathbb{T}_{1}$ and $\mathbb{T}_{2}^{\prime}=\pi\left(P_{2}\right)+\mathbb{T}_{2}$ be the asymptotic Mumford-Tate tori associated to $C_{1}$ and $C_{2}$.

Suppose $A$ is a product of two elliptic curves $E_{1} \times E_{2}$. Then there are some lattices $\Gamma_{1}$ and $\Gamma_{2}$ in $\mathbb{C}$ such that $E_{1}=\mathbb{C} / \Gamma_{1}$ and $E_{2}=\mathbb{C} / \Gamma_{2}$ and $\Gamma=\Gamma_{1} \times \Gamma_{2}$. Then $\widehat{\Gamma}=\widehat{\Gamma}_{1} \times \widehat{\Gamma}_{2}$.

We know by Lemma 3.2 that a character $\chi_{\theta}$ with

$$
\theta=\left(\theta_{1}, \theta_{2}\right) \in \widehat{\Gamma}=X^{*}(A)
$$

is trivial on $\mathbb{T}_{1}$ if and only if $\operatorname{Re}\left(\theta_{1} Z_{1}+\theta_{2} Z_{2}\right)$ is bounded on $C_{1}$. This is equivalent to saying that $\theta=\left(0, \theta_{2}\right) \in\{0\} \times \widehat{\Gamma}_{2}$. Then we have the exact sequence

$$
0 \rightarrow\{0\} \times \widehat{\Gamma}_{2} \rightarrow X^{*}(A)=\widehat{\Gamma}_{1} \times \widehat{\Gamma}_{2} \rightarrow X^{*}\left(\mathbb{T}_{1}\right)=\widehat{\Gamma}_{1} \times\{0\}=X^{*}\left(E_{1} \times\{0\}\right) \rightarrow 0 .
$$

As a consequence, the asymptotic torus $\mathbb{T}_{1}$ associated to $C_{1}$ is $E_{1} \times\{0\}$ and the same proof shows that the one associated to $C_{2}$ is $\{0\} \times E_{2}$. In this case,

$$
\overline{\pi(C)}=\pi(C) \cup E_{1} \times\{0\} \cup\{0\} \times E_{2} .
$$

If $\widehat{\Gamma}$ has no vector of the form $\theta=\left(0, \theta_{2}\right)$ with $\theta_{2} \neq 0$, then the only character of $A$ which is trivial on $\mathbb{T}_{1}$ is $\mathbf{1}_{A}$. In this situation, $\overline{\pi(C)}=A$. This is the generic situation.

It is also possible to construct examples of lattices $\Gamma \in \mathbb{C}^{2}$ where the set of $\theta \in \widehat{\Gamma}$ of the form $\theta=\left(0, \theta_{2}\right)$ is a rank one $\mathbb{Z}$-submodule of $\widehat{\Gamma}$. In this situation, $\mathbb{T}_{1}$ is a real torus of dimension 3 .

If there is no element $\theta \in \widehat{\Gamma}$ of the form $\theta=\left(\theta_{1}, 0\right)$ with $\theta_{1} \neq 0$, then $\mathbb{T}_{2}=A$ and $\overline{\pi(C)}=A$ but the sequence $\mu_{C, R}$ does not converge to $\mu_{A}$. In fact, by Theorem 2.4 , we have $\mu_{C, R} \rightarrow a \mu_{A}+b \mu_{\mathbb{T}_{1}}$ for some positive real constants $a$ and $b$.

If there is some $\theta \in \widehat{\Gamma}$ of the form $\theta=\left(\theta_{1}, 0\right)$ with $\theta_{1} \neq 0$, then $\mathbb{T}_{2}$ will be also a real torus of dimension 3 and

$$
\overline{\pi(C)}=\pi(C) \cup \mathbb{T}_{1} \cup \mathbb{T}_{2}
$$

6.3. Hyperelliptic curves in $\mathbb{C}^{2}$. Let $A$ be a complex abelian surface and let

$$
\pi: \mathbb{C}^{2} \rightarrow A=\mathbb{C}^{2} / \Gamma
$$

be the uniformizing map. Let $C$ be an hyperelliptic curve in $\mathbb{C}^{2}$ with equation

$$
Z_{2}^{2}=Z_{1}^{r}+a_{r-1} Z_{1}^{r-1}+\cdots+a_{0}
$$

for some integer $r \geq 3$ and some complex numbers $a_{0}, \ldots, a_{r-1}$.

Proposition 6.1. We have $\overline{\pi(C)}=A$ and $\mu_{C, R}$ converges weakly to the normalized Haar measure $\mu_{A}$ of $A$. 
Proof. For any branch $C_{\alpha}$ of $C$ we have

$$
\left|Z_{2}\right| \simeq\left|Z_{1}\right|^{\frac{r}{2}}
$$

Let $\mathbb{T}_{\alpha}^{\prime}=\pi(P)+\mathbb{T}_{\alpha}$ be the asymptotic torus associated to $C_{\alpha}$. We know by Lemma 3.2 that a character $\chi_{\theta}$ with $\theta=\left(\theta_{1}, \theta_{2}\right) \in \widehat{\Gamma}=X^{*}(A)$ is trivial on $\mathbb{T}_{\alpha}$ if and only if $\operatorname{Re}\left(\theta_{1} Z_{1}+\theta_{2} Z_{2}\right)$ is bounded on $C_{\alpha}$. This is only possible if $\theta_{1}=\theta_{2}=0$. Therefore $\mathbb{T}_{\alpha}=A$ and the result is a consequence of Theorem 2.4.

\section{References}

[1] J. Ax, On Schanuel's conjecture, Ann. of Math. (2) 93 (1971), 1-24.

[2] J. Ax, Some topics in differential algebraic geometry I: Analytic subgroups of algebraic groups, Amer. J. Math. 94 (1972), 1195-1204.

[3] B. Klingler, E. Ullmo and A. Yafaev, The hyperbolic Ax-Lindemann-Weierstrass theorem, Publ. Math. Inst. Hautes Études Sci., to appear.

[4] M. Orr, Introduction to abelian varieties and the Ax--Lindemann--Weierstrass theorem, in: O-minimality and diophantine geometry, London Math. Soc. Lecture Note Ser. 421, Cambridge University Press, Cambridge (2015), 100-128.

[5] J. Pila and J. Tsimermann, Ax-Lindemann for $\mathcal{A} g$, Ann. of Math. (2) 179, (2014), no. 2, 659-681.

[6] J. Pila and U. Zannier, Rational points in periodic analytic sets and the Manin-Mumford conjecture, Atti Accad. Naz. Lincei Cl. Sci. Fis. Mat. Nat. (9) Rend. Lincei Mat. Appl. 19 (2008), 149-162.

[7] E. M. Stein, Harmonic analysis, Princeton University Press, Princeton 1993.

[8] E. Ullmo and A. Yafaev, Hyperbolic Ax-Lindemann theorem in the cocompact case, Duke Math. J. 163 (2014), no. 2, 433-463

[9] R. Walker, Algebraic curves, Princeton University Press, Princeton 1950.

Emmanuel Ullmo, IHES, Laboratoire Alexander Grothendieck du CNRS, Université Paris-Saclay 35, route de Chartres, 91440, Bures-sur-Yvette, France e-mail: ullmo@ihes.fr

Andrei Yafaev, Department of Mathematics, University College London, Gower street WC1E 6BT, London, United Kingdom e-mail: yafaev@math.ucl.ac.uk

Eingegangen 17. Januar 2015 